\title{
Scaling in currency exchange
}

\author{
S. Galluccio ${ }^{\text {a, } *}$, G. Caldarelli ${ }^{\text {b,c, }, ~}$, M. Marsili a , Y.-C. Zhang ${ }^{\text {a }}$ \\ a Institut de Physique Théorique, Université de Fribourg Pérolles, Fribourg CH-1700, Switzerland \\ b S.I.S.S.A.II.S.A.S., v. Beirut 2-4, 1-34014 Grignano di Trieste (TS), Italy \\ c I.N.F.M., Istituto Nazionale di Fisica della Materia, Sezione di Trieste, Italy
}

Received 23 January 1997

\begin{abstract}
We study the scaling behavior in currency exchange rates. Our results suggest that they satisfy scaling with an exponent close to 0.5 , but that it differs qualitatively from that of a simple random walk. Indeed price variations cannot be considered as independent variables and subtle correlations are present. Furthermore, we introduce a novel statistical analysis for economic data which makes the physical properties of a signal more evident and eliminates the systematic effects of time periodicity.
\end{abstract}

PACS: 02.50.-r; 87.10.+e

Keywords: Scaling theory; Random walks; Diffusion processes

\section{Introduction}

Economy can be regarded as a many-body, complex system. From this point of view, it has been recognized that systems like stock exchange markets display scaling properties similar to those of systems in statistical physics. After the early works of Mandelbrot [1], the Boston group has studied in detail the scaling behavior of stock market indices, e.g. the Standard \& Poors 500 [2] and the Italian M.I.B. [3], as well as of indicators describing the growth of companies [4].

In the present paper we address the same problem with respect to the foreign exchange market (FX). This system, as we shall see, differs qualitatively from the stock exchange (SE) market. The Olsen group has recently carried out a parallel statistical analysis and found new and interesting results [5]. The first studies of intra-daily data [6-8], revealed a rich and complex behavior which cannot be fully understood in terms

\footnotetext{
* Corresponding author. E-mail: stefano.galluccio@unifr.ch.

${ }^{1}$ Present address: Department of Physics, The University of Manchester, Manchester M13 9PT, UK.
} 
of standard ARCH-GARCH stochastic models. Self-similarity and fractal structure of financial signals have been already discussed in some detail, as well [5].

Despite past extensive work [9-12], it is still unclear how to fully characterize the mathematical structure of the distributions of index returns and/or foreign exchange variations in real situations. Recently, it has been proposed that the dynamics of a foreign exchange market is characterized by a flow of statistical information from large scales to smaller ones [13]. The observations led to the conclusion that traders acting on the market at large time scales influence the traders acting more frequently on the market in a sort of "information cascade". Ghashghaie et al. [14] have further pushed this analogy by arguing that a financial market has some features in common with a turbulent hydrodynamic system in which the role of the statistical information is played by the energy released from large to small length scales in the well-known cascade process.

These authors had access to the same financial data we have also studied (from Olsen \& Associates Company) and they support their claim by comparing financial data with data obtained from a turbulent signal. In particular, they have found that the moments of the price deviations $\left\langle|\Delta P|^{m}\right\rangle$ do deviate from the standard scaling (such as real turbulence deviates from Kolmogorov's law).

However, Arneodo et al. [15] have pointed out that this analogy has only formal character, since the two situations substantially differ for the point of view of the correlations. While in hydrodynamic turbulence, the presence of long range velocity correlations leads to the usual power-law spectrum, financial data do not have significant correlations in time, and the disappearing of the distribution tails for large time scales is nothing but a consequence of the central limit theorem which still applies if the variables are weakly correlated in time. The same analogy between economics and turbulence, with similar conclusions, has been recently discussed by Mantegna and Stanley for the case of the Stock Exchange Index variations [16]. FX rates have also been analyzed in Ref. [17]. However, we shall deal with high-frequency data covering one year, which has been made available only recently [18], whereas in Ref. [17] data are analyzed over almost a century.

In our work, we further analyze the dynamics of a FX market from the point of view of temporal correlations and we find that subtle correlations do exist in the signal, as the system has memory also on large time scales.

In addition to this, we shall present a novel statistical analysis. This is motivated by the fact that the time series of FX rates cannot be considered as processes with stationary increments. Indeed, they suffer from systematic effects mainly due to the periodicity of human activities. It seems not obvious to us that the usual scaling analysis is still appropriate in this case. We have therefore introduced a novel time transformation to eliminate these systematic effects. A similar analysis was independently carried out by the Olsen group to eliminate problems due to periodic "seasonality" in the time signal. As in our case, in Ref. [19] it is argued that in order to avoid problems caused by the geographical dispersion of market agents, the time signal must be first filtered once the characteristic opening and closing times of different markets are determined. The central idea is the use of the volatility as an indicator of the activity in the market. A high 
mean volatility can be used to "expand" times when the activity on the market is large and "shrink" them in the opposite situation. In what follows we will use a similar analysis on our data and we discuss differences and similarities with the previous approach.

The data, obtained by the Olsen \& Associates Research Institute, refer to FX rates between US Dollar (USD), German Mark (DM) and Japanese Yen (JPY) from 1 October 1992 to 30 September 1993 . Hereafter, the currency price will be generally denoted as $P(t)$.

The data come in files where GMT time, and FX rates are reported sequentially. The number of data considered is 159079 for JPY-DM; 570814 for JPY-USD and 1472241 for USD-DM. They correspond to an average time between two consecutive transactions of 198.2, 55.2 and $21.4 \mathrm{~s}$, respectively, and for each currency we analyzed both bid and ask quotations recorded at consecutive times. These average values have been obtained considering the whole year with week ends and nights. We obtained the same results both considering the time series with the bid or the ask quotations. As in Ref. [19], data are international quotations of foreign currencies available from Reuters, Knight-Ridder and Telerate, and do not correspond to real prices in the global market.

We shall first discuss the results obtained with the usual, real-time statistics, and then move to the novel-time analysis. In what follows, we will consider the statistics of the currency exchange between DM and USD; numerical findings are qualitatively the same with other currency combinations.

\section{Real-time analysis}

In order to deal with a continuous signal we have shifted all the transaction times setting the time origin $(t=0)$ at the midnight of 1 October 1992. With linear interpolation, we assigned a value $P(t)$ for each time $t=n \delta t$ where the time unit chosen was of $\delta t=30 \mathrm{~s}$. In this way we defined the FX rates even in time periods, such as nights or week-ends, where no transaction was actually taking place. This choice differs from the one given in Refs. [2,3], where nights and week-ends were "cut" from the signal by assuming a time lag of a time step between the closure and the re-opening of the market. The reason of our choice is that the data were collected from several banks all around the world and therefore it was not possible to identify the closure and the re-opening of the FX market in an unique way.

With the above definition of time, we clearly need to define an error for the data in order to assign a more significant weight to those data which correspond to periods with a high level of activity.

The natural choice for the error on $P(t)$ is

$$
\sigma^{2}(t)=\left(\frac{1}{t-t_{0}}+\frac{1}{t_{1}-t}\right)^{-1} .
$$

Here $t_{0}$ and $t_{1}$ are two consecutive transaction times such that $t_{0}<t<t_{1}$. Eq. (1) implies that the variance of the data is inversely proportional to the density of points at that time. Therefore, Eq. (1) assigns a very small weight to data in the weekends. 

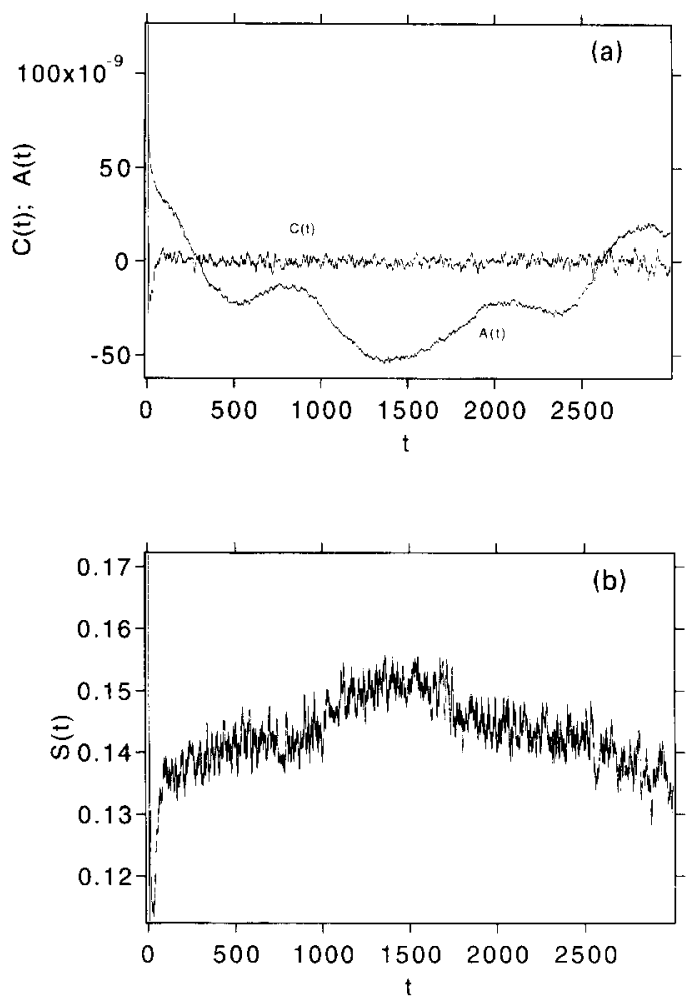

Fig. 1. (a) Correlation functions $C_{\tau}(t)$ and $A_{\tau}(t)$ for $\tau=8$ (DEM $\Rightarrow$ USD). (b) Sign correlation function $S_{8}(t)$. The time unit is fixed at $30 \mathrm{~s}$.

Errors on more complex quantities are computed by elementary statistics from $\sigma^{2}(t)$. For example, the error for the price difference

$$
\Delta P_{\tau}(t) \equiv P(t+\tau)-P(t)
$$

is $\sigma^{2}\left[\Delta P_{\tau}(t)\right]=\sigma^{2}(t+\tau)+\sigma^{2}(t)$. The average $\langle q(t)\rangle$ of a quantity $q(t)$ is computed using the weights $1 / \sigma^{2}[q(t)]$ in the time average.

The correlation function

$$
C_{\tau}(t) \equiv\left\langle\Delta P_{\tau}(t) \Delta P_{\tau}(0)\right\rangle-\left\langle\Delta P_{\tau}(0)\right\rangle^{2}
$$

(for $\tau=240 \mathrm{~s}$ ) is plotted versus $t$, in Fig. la. There are clearly no signs of correlation. The absence of correlations in the two point functions can be taken as evidence for the hypothesis of independent price variations [2,3]. This is, in a sense, a "strong" version of the no arbitrage hypothesis. The latter says that the time series $P(t)$ must be such that there cannot be any strategy, involving no risk, which allows to bargain from transactions. This clearly holds if $P(t)$ has independent increments, but it may hold under much more general conditions. 


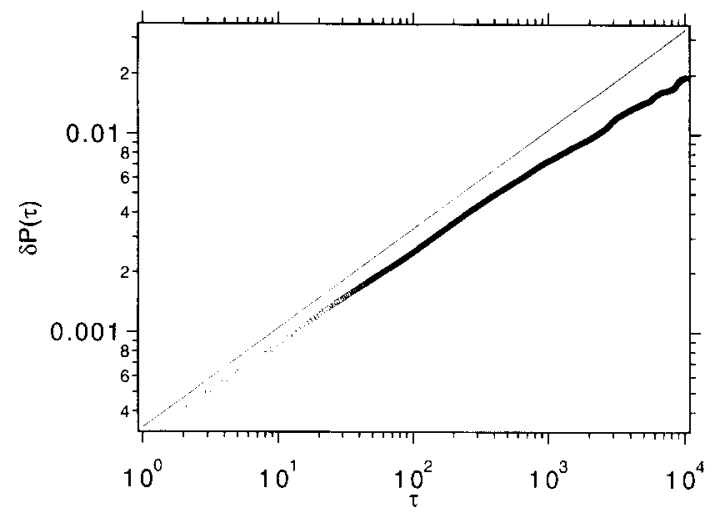

Fig. 2. Price variance $\delta P(\tau)$ versus $\tau$ from DEM $\Rightarrow$ USD data. The straight line corresponds to $\tau^{0.5}$.

In our case, FX rates do not have independent increments. This can be seen from the correlation function of the absolute value of price variations

$$
A_{\tau}(t) \equiv\left\langle\left|\Delta P_{\tau}(t) \| \Delta P_{\tau}(0)\right|\right\rangle-\left\langle\left|\Delta P_{\tau}(0)\right|\right\rangle^{2},
$$

and from the sign correlation function

$$
S_{\tau}(t) \equiv\left\langle\operatorname{sign}\left[\Delta P_{\tau}(t)\right] \operatorname{sign}\left[\Delta P_{\tau}(0)\right]\right\rangle-\left\langle\operatorname{sign}\left[\Delta P_{\tau}(0)\right]\right\rangle^{2},
$$

which are both shown in Fig. 1 .

The main result is that no correlation seems to exist between data except for the sign correlation function. It is worth pointing out that the signal is clearly periodic: the time period, of about 2900 units in Fig. 1, roughly corresponds to one day; the time unit is indeed of $30 \mathrm{~s}$. This effect is a consequence of human activities around the world and is a systematic effect in the signal.

We have also considered the average price variation

$$
\delta P(\tau)=\sqrt{\left\langle[P(t+\tau)-P(t)]^{2}\right\rangle}
$$

whose dependence on $\tau$ is plotted in Fig. 2. The slope of the function, in double logarithmic scale, suggests that

$$
\delta P(\tau) \sim \tau^{H}
$$

with a scaling exponent $H \simeq 0.45$.

Finally, we computed the histograms $F(x, \tau)$ of price variations $x=P(t+\tau)-P(t)$ for all currencies for several values of $\tau$. For DEM-USD exchange, these functions are shown in Fig. 3. We checked the scaling hypothesis

$$
F(x, \tau)=\tau^{h} F\left(\tau^{h} x, 1\right)=\tau^{h} g\left(\tau^{h} x\right)
$$

by plotting $F(x, \tau) / \tau^{h}$ versus $\tau^{h} x$ and the best collapse was obtained for $h=0.5$. 

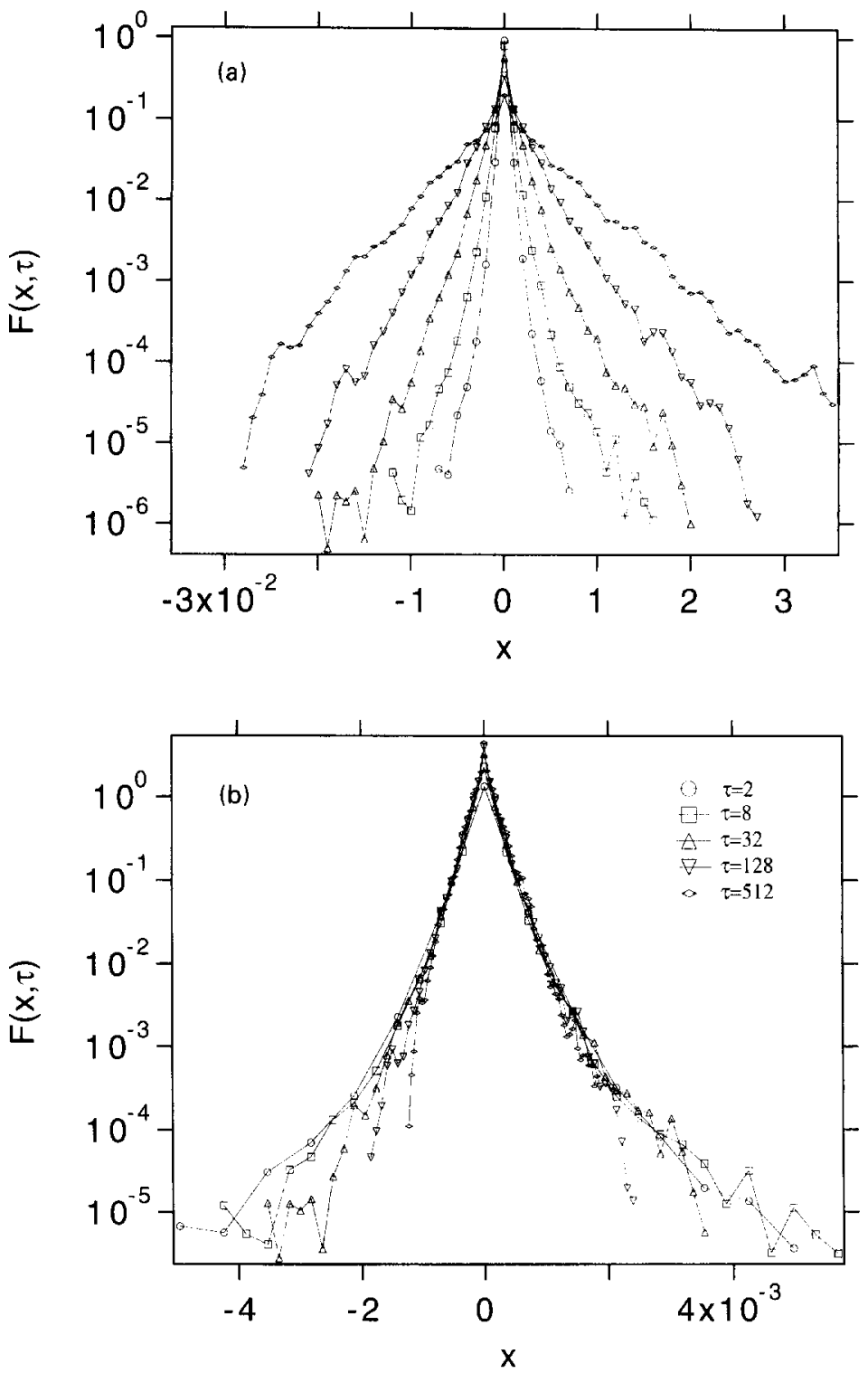

Fig. 3. (a) Histograms of price variations. (b) Collapsed distributions with $H=0.5$ (DEM $\Rightarrow$ USD).

The best fit was given by a stretched exponential form $F(x) \sim \exp \left(-|x|^{\beta}\right)$, with $\beta=$ $1.10 \pm 0.02$, for small $x$ and algebraic tails $F(x) \sim|x|^{-\omega}$ with $\omega=5.0 \pm 0.5$, for large $x$. As in Ref. [14] we have found a slight change of the distribution shapes in passing from low to high time scales. In particular, it seems that by increasing the time interval $\tau$ the distribution densities tend to become gaussians. In Ref. [15], a similar study was performed in order to detect the presence of correlations and the structure of the distribution functions. The authors find that apart from the tails, the distributions seem 
to follow a Lévy stable law, as for the stock markets index variations [2]. Due to the lack of a good statistics on the data, we believe, however, that the problem of establishing the mathematical origin of these distributions is still far to be solved. Our numerical data did not allow to check whether scaling holds far in the tails of the distribution. This would indicate whether the markets react the same way in presence of small or big price fluctuations.

Moreover, we have found a scaling exponent $H \simeq 0.45$ which is quite different from the one observed for the SE indicators $(H \simeq 0.65[1-3])$. Generally speaking, $H$ and $h$ need not to be the same. Even though our results seem to suggest that they are close to each other, they are not conclusive, due to a lack of more significant data. In Ref. [20] a similar test was performed on price changes. Instead of considering the statistics of the price difference $P(t)$, the authors considered the signal defined by the logarithmic price change $Q(t)=\left[\log \left(p_{\text {bid }}(t)\right)+\log \left(p_{\text {ask }}(t)\right)\right] / 2$. This is motivated by the fact that, in general, financial time series are supposed to be associated to multiplicative dynamical processes. For this class of systems, the statistics of the logarithm of the increments is surely the most appropriate quantity of investigation. For high-frequency data, the relative increments of the signal between consecutive times are small and no significative differences are supposed to exist if one performs the statistical analysis with linear increments.

Moreover, in Ref. [20], the dependence of the average of $|Q(t)|$ on time was studied and a power-law behavior with exponent $D \sim 0.586$ was found. This result was shown to be independent from the particular combination of currencies one takes into account. The apparent disagreement between our value of $H$ and $D$, is due to the presence of multiscaling in the temporal signal. The first moment can indeed show a different scaling with time. In fact, if the time increments are distributed according to a multifractal density, or equivalently, if the distribution of price changes presents different scaling for different time intervals, the first moment $\langle|P(t)|\rangle$ is larger than $\left\langle(P(t)-\langle P(t)\rangle)^{2}\right\rangle^{1 / 2}$. This is due to the convex property of the $m$-order moments $\zeta_{m}$ (see later) as functions of $m$ [21]. Price changes are actually distributed according to a multifractal density, as we will show in the next section.

\section{Intrinsic time analysis}

The statistical analysis performed in the previous section is tailored for processes with stationary increments. The signal $P(t)$ is not at all of this kind. This is evident if one measures the standard deviation of $P(t)$ as a function of the real time in the interval corresponding to a week. More precisely let $\overline{P(t)}$ be the average price over a short time interval $\delta t$ (typically of the order of half a hour) around $t$. Let us define the volatility

$$
V(t)=\left\langle\overline{[P(t)-\overline{P(t)}]^{2}}\right\rangle
$$

as the variance of the price over the same time interval. 


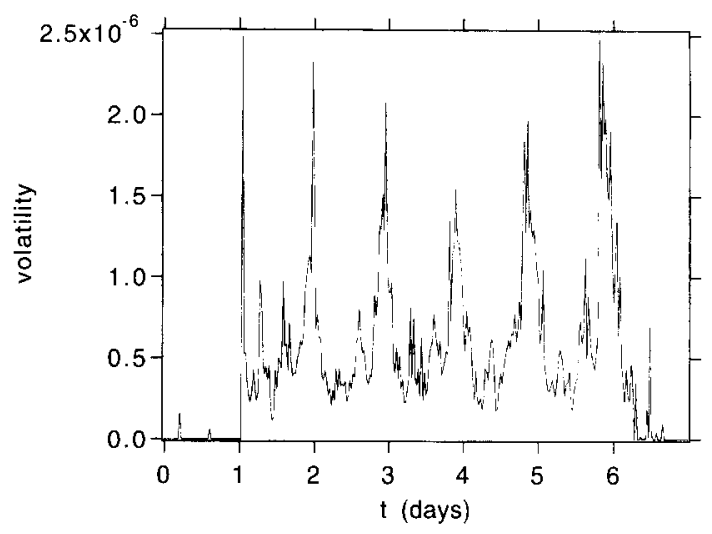

Fig. 4. Volatility of the DEM-USD exchange rate in one week, averaged over all the weeks in one year.

A plot of $V(t)$ for $t$ spanning one week (Fig. 4), averaged over all the weeks of the year displays variations which are clearly periodic with a period of 1 day. If one imagines that the signal $P(t)$ is produced by a system of $N$ agents, it is most likely that the systematic periodicity of $P(t)$ results from an analogous periodicity of $N(t)$ in real time.

It is desirable to get rid of these systematic effects for various reasons. First, as mentioned above, it is not clear whether in their presence the scaling analysis is still meaningful. Indeed, it has been always applied in statistical physics to processes with stationary time increments. For these processes, the presence of scaling is equivalent to the statement that there is no characteristic scale in the system (a characteristic time, in our case). This statement strongly contrasts with the presence of time-scales associated with days, weeks and months which are present in economic data. Once these systematic effects are washed out, one may hope that the statistical properties of the signal can emerge more clearly.

Secondly, a process with stationary increments is surely more appealing from a theoretical point of view. In statistical physics the number of degrees of freedom $N$ is usually fixed as an external parameter. It is highly desirable, if one wants to model an economic system, to have a reference analysis where the systematic effects of time periodicity have been filtered out.

In order to eliminate the periodicity of the signal $P(t)$, we define an intrinsic, or "inner" time, as

$$
s(t)=\int_{0}^{t} V\left(t^{\prime}\right) \mathrm{d} t^{\prime},
$$

where the volatility is taken on a short time interval of $30 \mathrm{~s}$. The new series of data $s\left(t_{i}\right), P\left(t_{i}\right)$ is then interpolated to obtain $B(s)=P(t(s))$ for $s=n \delta s$. $\delta s$ was chosen in such a way as to have the same statistics as before. 

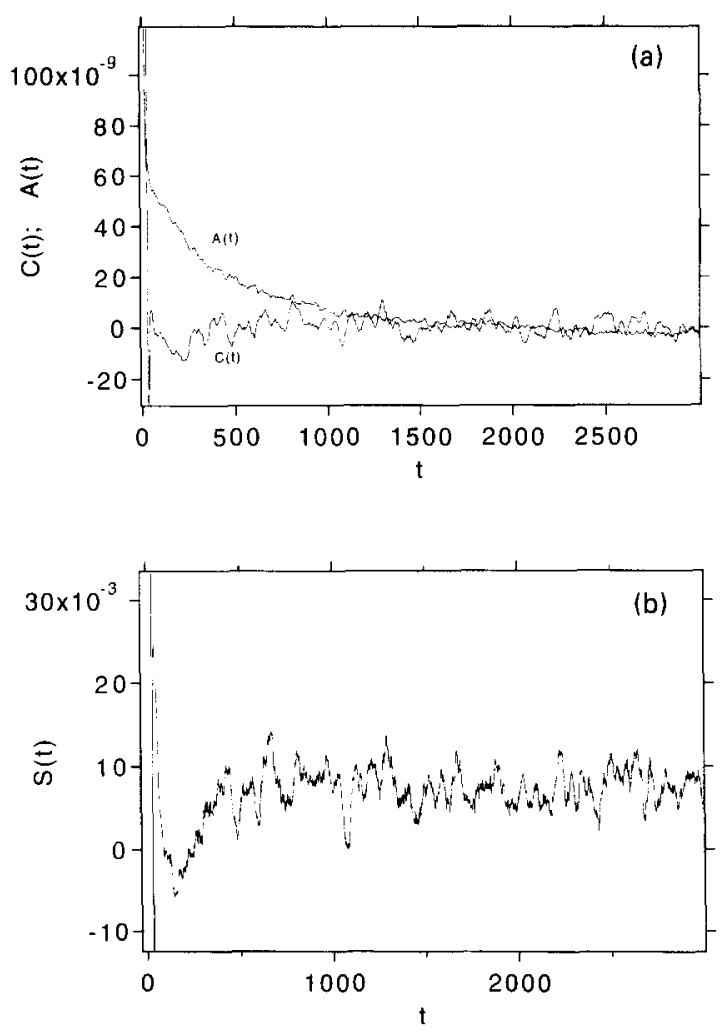

Fig. 5. (a) Correlation functions $C_{\tau}(t)$ and $A_{\tau}(t)$ for $\tau=8$ (DEM $\Rightarrow$ USD) with intrinsic time analysis. (b) Sign correlation function $S_{8}(t)$.

It is worth to point out that the above time transformation would leave $P(t)$ invariant if $P(t)$ were a simple random walk. In a loose sense, $V(t)=\mathrm{d} s / \mathrm{d} t$ is a measure of the activity of the FX market at time $t$. In the new time the dynamics is "at constant activity". Our transformation is in the same spirit of the observation [22] that economic data can be modeled as a process with increments given by a probability distribution $p\left(x / \gamma_{t}\right) / \gamma_{t}$ where $\gamma_{t}$ is a time-dependent scale factor.

Finally, note that with our redefinition of time we integrate the fluctuations only on a small time-scale. Therefore, if scaling holds on a range of time-scales above this scale, we expect to see it even in the "inner" time statistics.

In Figs. 5 and 6, we report $C_{\tau}(s), A_{\tau}(s), S_{\tau}(s)$ and $\delta P(\tau)$ in the intrinsic time. A comparison with Figs. 1 and 2 shows that the statistical properties are much cleaner in the former ones. There is no more trace of periodicity in the absolute value correlation function $A(t)$ and, as it is evident from Fig. 5, it shows a rather slow convergence to 0 , characterized by an exponential decaying $A_{\tau}(s) \sim \exp (-s / S)$ with $S \simeq 1.34 \times 10^{4}$, roughly corresponding to $3.74 \mathrm{~h}$ in real time. This suggests that price variations are indeed correlated up to a time interval of the order of $4 \mathrm{~h}$ in average. Clearly, in view 


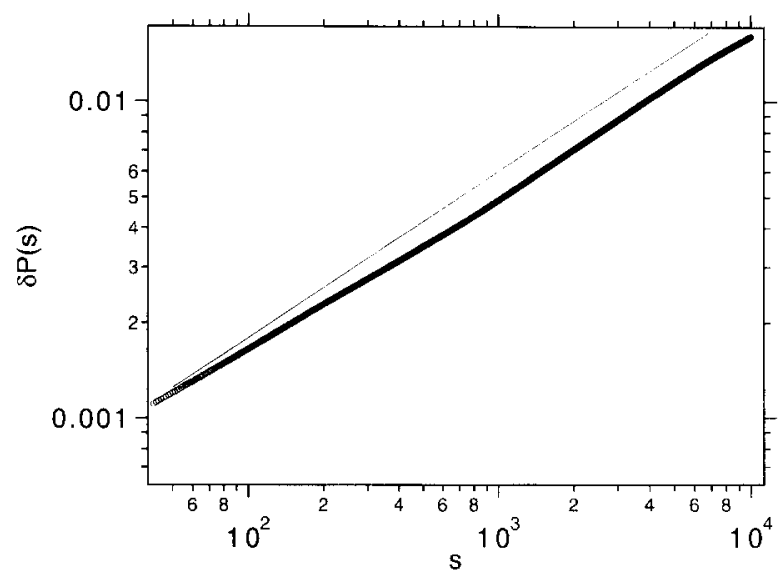

Fig. 6. Price variance $\delta P(\tau)$ versus $\tau$ from DEM $\Rightarrow$ USD data with intrinsic time statistics. The straight line corresponds to $\tau^{0.5}$.

of the definition of $S(t)$, periods with high level of activity have a smaller characteristic "real" time.

Moreover, from Fig. 6, we can establish that the scaling exponent in the new time is $H_{s}=0.50 \pm 0.01$ with much more accuracy. Even though this exponent is the same of that of a random walk, it is important to stress that this is not an artifact of the time transformation. Indeed, with Ref. [17], we believe that the scaling properties are related to the persistence properties of the signal $P(t)$ and not to the statistics of single time increments. The persistence is not affected by our transformation. In order to check this point, we generated random fractional walks $[23,24]$ with a preassigned value of the Hurst exponent $H$. The scaling of these walks was then analyzed with respect to their "inner" time with the result that $H_{s}=H$. This suggests that the systematic effects of periodicity significantly affect the evaluation of the scaling exponents in real time.

In Fig. 7, we show the histograms of the price differences in the "inner" time. These distributions seem to collapse quite well at $h_{s}=0.5$ for $\tau \in[2,512)$. The qualitative behavior of these distributions is very similar to that of the real-time analysis.

Similar ideas were independently introduced to eliminate seasonality in financial time series by the Olsen group [16]. The only significant difference with our approach is the definition of the transformation to the "inner time". Instead of Eq. (3), the authors in Ref. [19] consider the absolute price change in a given interval $t, V(t)=\mid P(\tau)-P(t+$ $\tau) \mid$, as their definition of volatility. As a consequence, the time transformation Eq. (4) must be accordingly modified. The time transformation based on the second moment is statistically more meaningful and natural. Indeed our Eqs. (3) and (4), applied to the paradigm of a simple random walk, give exactly the "inner time" of the RW itself. This would not hold if $V(t)=|P(\tau)-P(t+\tau)|$, since a linear dependence of $V(t)$ on $t$ is necessary to do the job. 

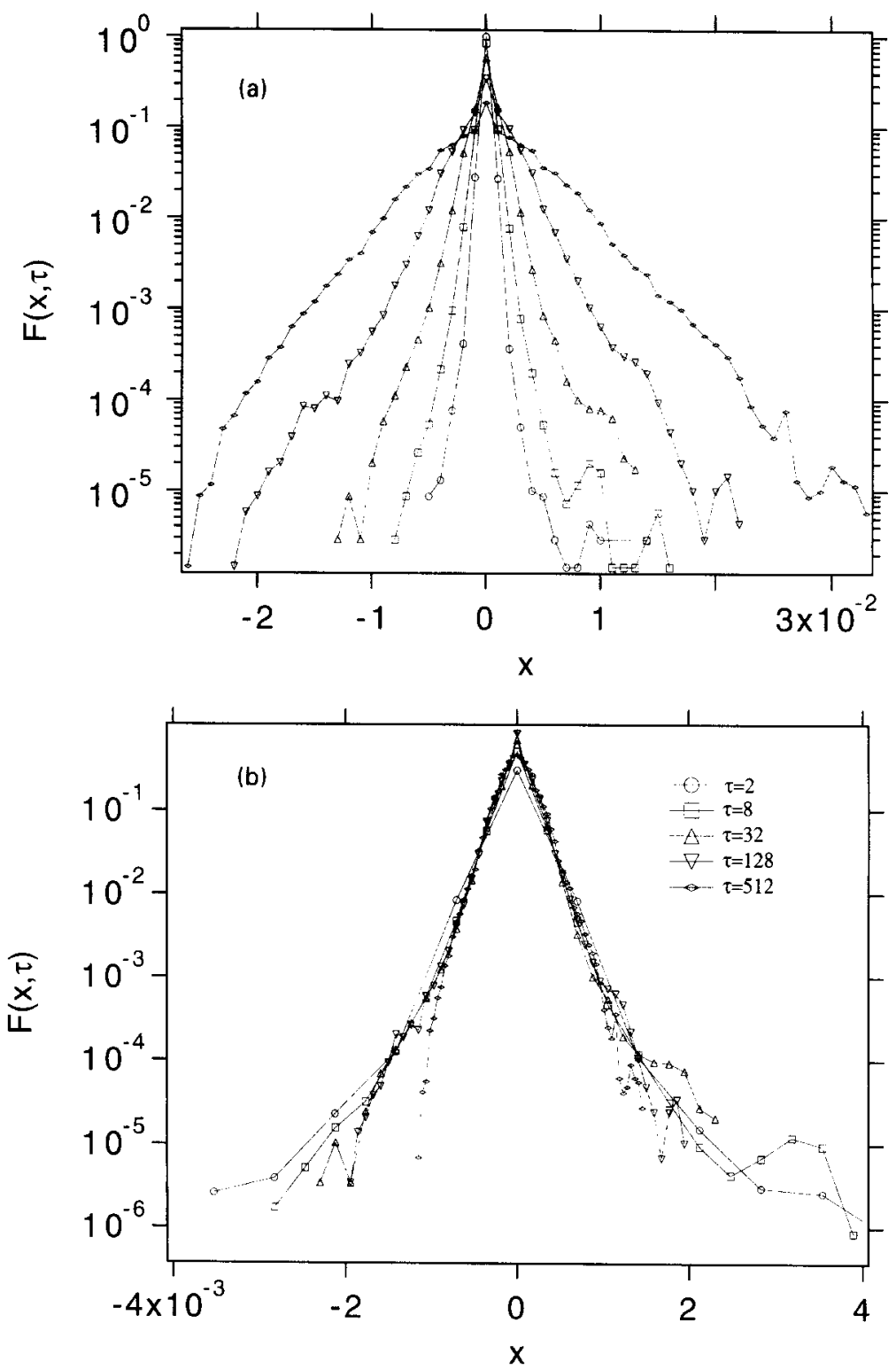

Fig. 7. (a) Histograms of price variations with intrinsic time statistics. (b) Collapsed distributions with $H=0.5$ (DEM $\Rightarrow$ USD).

In order to prove that the two approaches are not equivalent, we can compare the correlation function $A(t)$ in Fig. 5, with the equivalent computation performed in Refs. $[5,19]$ in the rescaled time. Instead of the exponential decay we described previously, the authors claim that $A(t)$ approaches 0 with power-law tails at large times. Therefore their results seem to indicate that the absolute value of price changes is significantly correlated in time even for intervals of many days apart. If this were true one could 


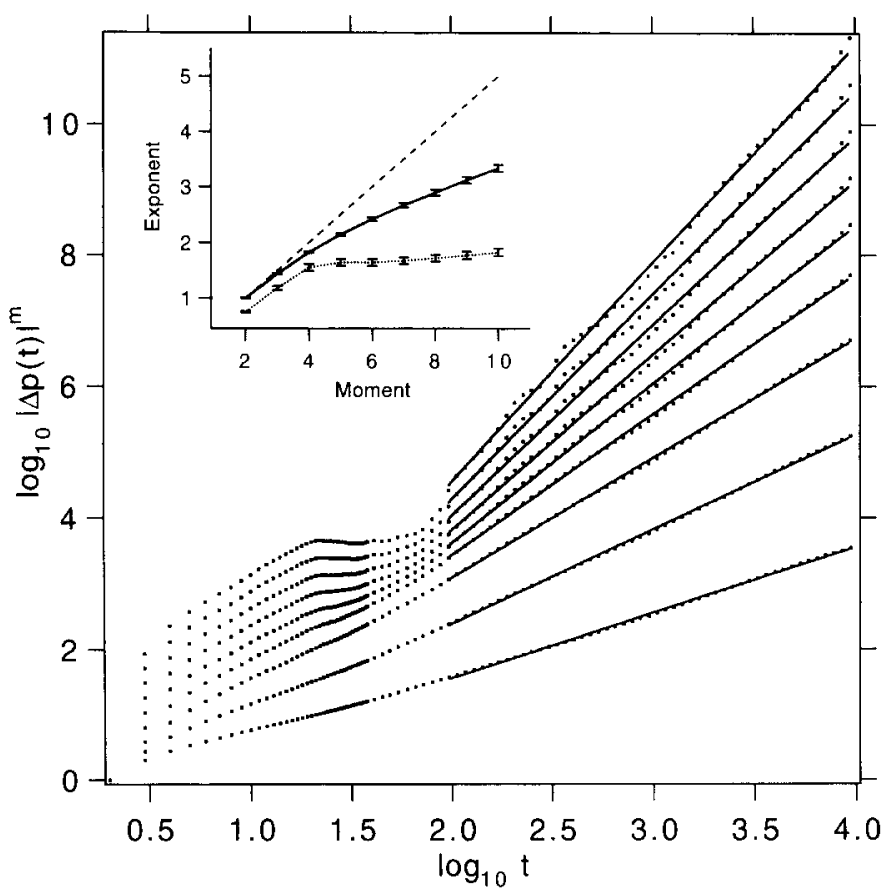

Fig. 8. The moments of the price variations $\zeta_{m}=\left\langle|\Delta P|^{m}\right\rangle$. The slopes of the fitting lines are our estimates of $\zeta_{m}$, for $m=2, \cdots, 10$. In the inset $\zeta_{m}$ versus $m$ is shown. Note the deviation from a simple random walk scaling, represented by the dashed straight line. The lower curve refers to the exponents $\xi_{m}$ in the range $2<\tau<20$.

easily take advantage of this large correlation in order to formulate long-time forecasts in the currency quotations.

Finally, we carried out analysis similar to the one presented in Ref. [14] to detect the presence of multiscaling. For the real-time statistics we confirmed the results of Ref. [14] whereas the results of our analysis with "inner" time are displayed in Fig. 8. As it can be clearly seen, the moments $\zeta_{m}=\left\langle\left|\Delta P_{\tau}\right|^{m}\right\rangle$ have power-law behavior,

$$
\zeta_{m} \simeq \tau^{\xi_{m}}, \quad 10^{2}<\tau<10^{4}
$$

The scaling exponents $\xi_{m}$ do not display the typical behavior of "gap scaling", for which $\xi_{m}=a+b m$. Instead, they seem to bend continuously approaching the line $\xi_{m} \simeq 1.1+0.22 m$ as $m \rightarrow \infty$. Let us note that the value of $b=0.22$ roughly corresponds to the inverse of the exponent $\omega-1 \simeq 4.0 \pm 0.5$ which characterizes the power-law behavior of the tail of the distribution function. Remarkably, this is the result one would obtain for the largest among $\tau$ variables which are distributed according to $F(x) \simeq|x|^{-\omega}$ for $|x| \gg 1$.

The implications of multiscaling in FX rates have already been discussed in Ref. [14] and we will not address the same problem here. The point we wish to stress is that this feature emerges much more manifestly in the intrinsic time analysis than in 
the real-time one. We can also identify an early regime (for $\tau=\Delta s<20$ ) where the signal presents an even stronger multiscaling with the exponent $\xi_{m}$ which quickly saturates to $\xi_{m} \simeq 1.8$. Instead, for $\tau>10^{4}$, which roughly corresponds to the time correlation time previously discussed, the multiscaling behavior seems to crossover to a standard random walk scaling.

\section{Conclusions}

The dynamics of large correlated systems in nature exhibits a high level of complexity. This is due to the effect of correlations between individuals (or particles) resulting in a collective behavior. Examples include self-organized criticality in physics and dynamics of financial markets in economy. From both the theoretical and the practical points of view, in the study of FX markets, the understanding of the statistical nature of the variables which are involved is of big interest.

We have carried out a statistical analysis on data concerning currency exchange among US Dollar, Japanese Yen and German Mark both in real time and using a novel transformation to avoid problems due to the non-stationarity of the temporal signal.

Hidden correlations are present in the data and we have found that in the "inner" time statistics, in which the signal has stationary increments, the price variations scale in time with Hurst exponent $\frac{1}{2}$. Even though this exponent coincides with that of a simple random walk, we found that FX rates have a far more complex nature. First we found that the sign correlation function and the absolute value correlation function show that correlations are present in the system. Secondly, as pointed out in Ref. [14], FX rates show multiscaling behavior.

Both these features can be observed in the real-time statistics, but they emerge in a clearer way in the "intrinsic time" analysis. This suggest that the non-stationarity of the signal does affect the results of a statistical analysis and it might lead to unprecise or even wrong conclusions.

The "inner" time transformation provides a simple tool by which a consistent statistical study can be carried out for these complex systems.

\section{Acknowledgements}

The authors acknowledge Olsen \& Associates for providing the FX data.

\section{References}

[1] B.B. Mandelbrot, J. Business 36 (1963) 394; J. Bus. University of Chicago 39 (1966) 242; J. Bus. University of Chicago 40 (1967) 393.

[2] R.N. Mantegna, H.E. Stanley, Nature 376 (1995) 46.

[3] R.N. Mantegna, Physica A 179 (1991) 232. 
[4] M.H.R. Stanley, L.A.N. Amaral, S.V. Buldyrev, S. Havlin, H. Leschhorn, P. Maass, M.A. Salinger, H.E. Stanley, Nature 379 (1996) 804.

[5] U.A. Müller, M.M. Dacorogna, R.D. Davé, O.V. Pictet, J.E. Wizsäcker, Fractals and intrinsic time -a challenge to econometricians, invited presentation at the XXXIXth International AEA Conference on Real Time Econometrics, Luxembourg, 1993; UAM 1993-08-16, Olsen \& Associates, Zürich, Switzerland.

[6] R.T. Baillie, T. Bollersev, Rev. Economic Studies 58 (1990) 565.

[7] R.F. Engle, T. Ito, W.-L. Lin, Econometrica 58 (1990) 525.

[8] C.A.E. Goodhart, L. Figliuoli, J. Int. Money and Finance 10 (1991) 23.

[9] M.F.M. Osborne, Oper Res. 7 (1959) 145.

[10] E.F.J. Fama, J. Bus. 38 (1965) 34.

[11] R.F. Engle, Econometrica 50 (1982) 987.

[12] T. Bollersev, R.Y. Chou, K.F.J Kroner, Econometrics 52 (1992) 5.

[13] U.A. Müller, M.M. Dacorogna, R. Davé, R.B. Olsen, O.V. Pictet, J.E. von Weizsäcker, J. Empirical Fin., 4 (1997) 213.

[14] S. Ghashghaie et al., Nature 381 (1996) 767.

[15] A. Arneodo, J.P. Bouchaud, R. Cont, J.F. Muzy, M. Potters, D. Sornette, Comment on 'Turbulent Cascades in Foreign Exchange Markets', Nature, submitted; cond-mat. preprint 9607120.

[16] R.N. Mantegna, H.E. Stanley, Stock Market Dynamics and Turbulence: Parallel Analysis of Fluctuation Phenomena, Boston University preprint.

[17] E.E. Peters, Fractal Market Analysis, Wiley, New York, 1994.

[18] Financial data were obtained by Olsen \& Associates International Company. Internet address: http://www.olsen.ch.

[19] M.M. Dacorogna, U.A. Müller, R.J. Nagler, R.B. Olsen, O.V. Pictet, J. Int. Money and Finance 12 (1993) 413.

[20] U.A. Müller, M.M. Dacorogna, R.B. Olsen, O.V. Pictet, M. Schwarz, C. Morgenegg, J. Banking and Finance 14 (1990) 1189.

[21] G. Paladin, A. Vulpiani, Phys. Rep 156 (1987) 147.

[22] M. Potters, R. Cont, J.P. Bouchaud, cond-mat. preprint/9609172.

[23] B.B. Mandelbrot, J.W. Van Ness, SIAM Rev 10 (1968) 422.

[24] K. Falconer, Fractal Geometry, Wiley, New York, 1990. 